OPEN ACCESS

Edited by:

Eric D. Caine,

University of Rochester, United States

Reviewed by:

Eberhard A. Deisenhammer Innsbruck Medical University, Austria

Natalie Riblet,

White River Junction VA Medical

Center, United States

${ }^{*}$ Correspondence:

Lana Bojanic

lana.bojanic-2@manchester.ac.uk

Specialty section: This article was submitted to

Public Mental Health,

a section of the journal

Frontiers in Psychiatry

Received: 09 December 2019

Accepted: 18 May 2020

Published: 09 June 2020

Citation:

Bojanić L, Hunt IM, Baird A, Kapur N, Appleby L and Turnbull P (2020) Early

Post-Discharge Suicide in Mental Health Patients: Findings From a National Clinical Survey.

Front. Psychiatry 11:502. doi: 10.3389/fpsyt.2020.00502

\section{Early Post-Discharge Suicide in Mental Health Patients: Findings From a National Clinical Survey}

\author{
Lana Bojanić $^{1 *}$, Isabelle M. Hunt ${ }^{1}$, Alison Baird ${ }^{1}$, Navneet Kapur ${ }^{1,2}$, Louis Appleby ${ }^{1,2}$ \\ and Pauline Turnbull ${ }^{1}$ \\ ${ }^{1}$ National Confidential Inquiry into Suicide and Safety in Mental Health, Faculty of Biology, Medicine and Health, The \\ University of Manchester, Manchester, United Kingdom, ${ }^{2}$ Greater Manchester Mental Health NHS Foundation Trust, \\ Manchester, United Kingdom
}

Studies on suicide by recently discharged mental health patients have reported a high number of deaths in the early post-discharge period, which has led to recommendations of follow-up within 7 days (d). More recently, the National Confidential Inquiry into Suicide and Safety in Mental Health ( $\mathrm{NCISH}$ ) proposed a more "stringent" follow-up period of 2-3 days (d) after discharge. Patients who died within this early time-frame post-discharge were more likely to die before the follow-up appointment occurred. They more often had a primary diagnosis of a personality disorder, self-discharged, and had a higher frequency of death by jumping from a height or in front of the vehicle compared to later deaths. This study provides practical implications for post-discharge management and safety planning. Clinicians should be aware of (1) the increased risk of immediate suicide in the postdischarge period by people with a diagnosis of personality disorder, (2) immediate suicide risk in patients who initiate their own discharge, and (3) the increased risk of death by jumping from a height or in front of the vehicle in the immediate post-discharge period. Our findings support the recent recommendation from $\mathrm{NCISH}$ that follow-up should occur within $3 \mathrm{~d}$ of discharge from in-patient care.

Keywords: follow-up, self-discharge, suicide method, personality disorder, mental health patients, post-discharge

\section{INTRODUCTION}

Discharge from psychiatric in-patient care is a period of high risk for suicide (1). In the UK, postdischarge suicides make up $17 \%$ of all patient suicide deaths (2). A recent meta-analysis of suicide rates after discharge from psychiatric facilities suggests these rates remain high for several years (3), but are particularly high in the first few months $(4,5)$ and weeks $(6-8)$ post-discharge. These findings have led to a recommended follow-up period within 7 days (d) of discharge for all patients, and within $48 \mathrm{~h}$ if a risk of suicide has been identified (9). There is some evidence that this intervention has impacted suicide rates; the 2018 report from the National Confidential Inquiry into Suicide and Safety in Mental Health (NCISH) (2) reported a fall in suicide rates within 3 months post-discharge in England following a peak in 2011.

However, as Chung and colleagues (1) point out, this time frame still means that some patients are likely to die before their scheduled appointment. In a controlled study of 100 patient deaths by 
suicide within 2 weeks of discharge, Bickley and colleagues (6) reported that $55 \%$ occurred in the first week, $49 \%$ of whom died before their first follow-up appointment. More recently, NCISH reported the second and third day post-discharge to be the highest risk periods and suggested a more "stringent" followup within 3 days (d) of discharge (2).

A number of studies have examined the characteristics of patients who died by suicide shortly after discharge. The majority have reported higher rates of post-discharge suicide in men (1), with the exception of a study linking a Finnish nationwide register with in-patient data (10) which reported more female patients dying in the first week post-discharge. Studies also report being older than 40 (6), unmarried (10), unemployed (11), and living alone or having low levels of social support (1113) as risk factors for post-discharge suicide. In UK studies that have examined post-discharge suicide, the most common methods of suicide were hanging and self-poisoning (4-6). However, other international studies have reported more deaths by jumping from a height (12) and drowning (10), citing availability, and lethality of these methods. In respect of psychiatric diagnosis, post-discharge suicide is most commonly associated with diagnoses of affective disorders, namely depression, and schizophrenia $(4,10,11)$. Examination of linked Swedish registers (14) found a higher risk of suicide within a month post-discharge in male patients with a diagnosis of depression and a reaction to crisis in comparison to patients with other diagnoses.

A finding repeated across studies regardless of the postdischarge time frame is a higher risk in patients who initiated their own discharge $(4,5,7,15-17)$. Further clinical factors associated with suicide include non-adherence to treatment (4, $10,11)$, a short final admission $(5,6,16,18)$, and a history of/ current self-harm $(4,12)$. Findings from prospective studies have shown that suicidal ideation was more elevated in patients who died by suicide 1,3 , and 6 months after discharge compared to other discharged inpatients $(19,20)$. However, little is known about whether the characteristics of patients who die by suicide within the immediate post-discharge period, i.e. less than a week, differ from later post-discharge deaths (2). An awareness of the features of these patients could aid care and discharge safety planning. In this study we aim to examine factors associated with immediate suicide following discharge from psychiatric inpatient care, and any subsequent practical implications for the management of care. We describe the socio-demographic and clinical characteristics of patients who died by suicide in the first $3 \mathrm{~d}$ after discharge in comparison with those who died between 4 and $7 \mathrm{~d}$ post-discharge, and present the rates of early postdischarge suicides.

\section{MATERIALS AND METHODS}

\section{Data Collection}

NCISH collects data on all deaths by suicide by people in the United Kingdom who were in contact with mental health services in the 12 months prior to death (further referred to as "patients"). The method of the NCISH data collection is fully described elsewhere (21). In short, data collection starts with the identification of all cases of death by suicide including deaths with undetermined intent from the Office for National Statistics. Information on contact with mental health services in the 12 months before death is then obtained from the National Health Service (NHS) trusts and Health Boards in the deceased's district of residence. Lastly, clinical data is obtained via questionnaires completed by the patient's supervising clinician. The questionnaire collects information on socio-demographics, psychosocial history, life events, details of the suicide, treatment and adherence, and last contact with services (including the date of last discharge). The sample presented here consists of patients who died by suicide within a week of discharge from psychiatric in-patient care between January 1997 and December 2016 inclusive, based on the date of death. NCISH achieves a questionnaire response rate of $>95 \%$ (4). $\mathrm{NCISH}$ has research ethics approval from the North West Research Ethical Committee and approval under Section 251 of the NHS Act 2006 (originally Section 60 of the Health and Social Care Act 2001).

\section{Statistical Analysis}

In the present analysis we focused on England and Wales data due to the differences in service provision between devolved nations. Two groups of patients were examined based on the time of their death in relation to discharge: patients who died by suicide within $3 \mathrm{~d}$ post-discharge ( $3 \mathrm{~d}$ group) and those who died by suicide between the fourth and seventh day post-discharge (1 week group). We grouped patients who died on day 0 postdischarge (i.e. on the actual day of discharge) into the 3 d group (Figure 1). We have only included community patients who have been formally discharged and had a discharge date recorded. Since we do not record time of the death and time of the discharge we have calculated the time between death and the discharge as the difference in days.

Descriptive analysis (presenting the frequency and percentages) and comparisons (using chi-square tests) between the discharge groups were carried out. Descriptive statistics are presented as valid percentages, adjusting for the occurrences of missing data. We compared patients on various sociodemographic and clinical characteristics, as well as on suicide method, primary diagnosis, and estimation of suicide risk by the clinician. Results with $p<.05$ were considered to be statistically significant.

Rates were calculated for the years with complete numerator and available denominator data from NHS Digital, i.e. 20052015. Data for 2016 were incomplete due to the time associated with legal processes and were therefore omitted from the rate analysis. Denominators used were total number of mental health in-patient admissions and discharges in England and Wales obtained from NHS Digital which collects data on in-patient activity. Rates for the $3 \mathrm{~d}$ group have been adjusted to account for the discrepancy between the number of days between groups. Due to the numbers per year being small, in-depth time series analysis could not be performed. This was also prohibitive in terms of adjusting for other demographic variables such as age and gender. All analyses were carried out using statistical 


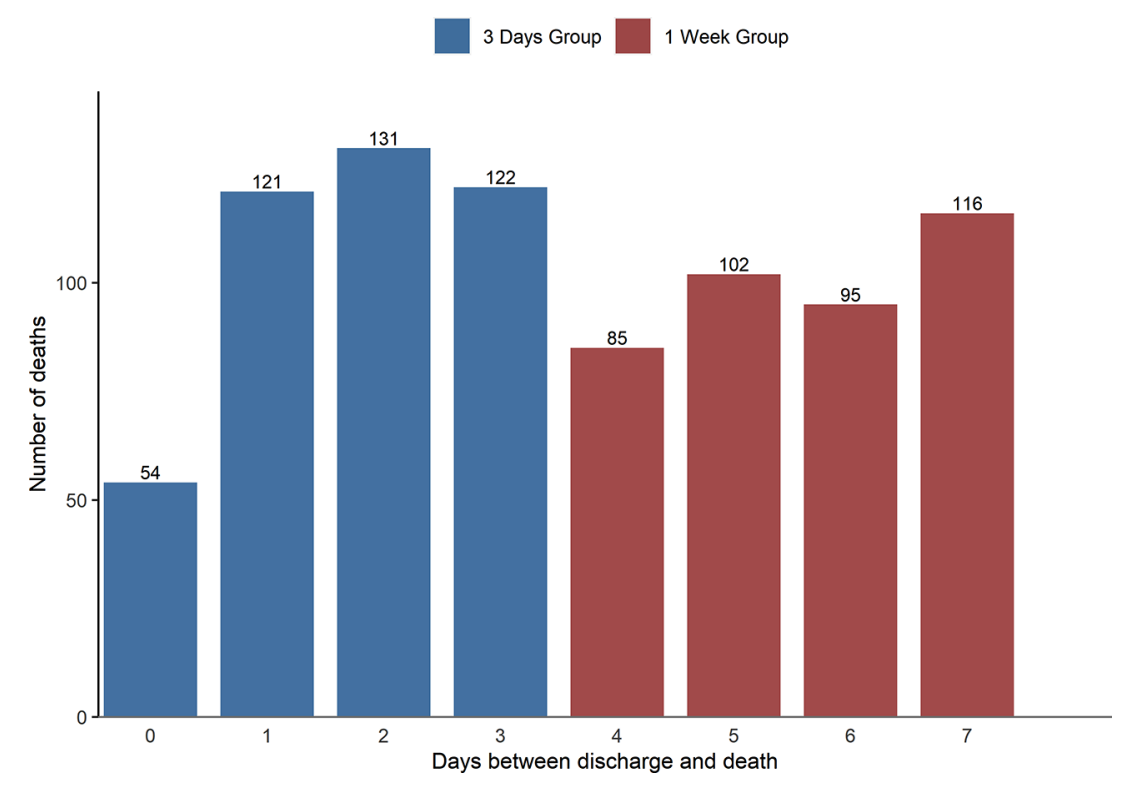

FIGURE 1 | Frequency of deaths by suicide in England and Wales between 1st January 1997 until 31st December 2016 by discharge day ( $N=826)$.

software STATA Version 15 (22). All the figures were produced using RStudio 3.4.3 (23).

\section{RESULTS}

\section{Descriptive Analysis and Rates}

Over the 20-year period from $1^{\text {st }}$ January 1997 until 31st December 2016, we were notified of 26,426 deaths identified as patient suicides (i.e. they had been in contact with mental health services in the 12 months before death). A total of 826 (3.1\%) died within a week of discharge from psychiatric inpatient care. Within this time frame, 428 (51.8\%) died within $3 \mathrm{~d}$ ( $3 \mathrm{~d}$ group) and 398 (48.2\%) died between 4 and $7 \mathrm{~d}$ (1 week group). Patients in the two groups did not differ in age (median in the $3 \mathrm{~d}$ group $=46$ (range 16-94) v. median in the 1 week group $=46$ (range 15-87), $\left.\chi^{2}=0.18, d f=1, p=.67\right)$. Frequencies of suicide deaths by days since discharge are shown in Figure 1.

Early post-discharge suicide rates by year and number of admissions and discharges, respectively, are presented in Figures 2 and 3. Overall rates for the $3 \mathrm{~d}$ group were 0.18 per 100,000 admissions and 0.19 per 100,000 discharges. Overall rates for the 1 week group were 0.14 per 100,000 admissions and 0.14 per 100,000 discharges. Rates per admissions and discharges generally followed the same pattern. Despite fluctuations, rates were slightly higher for the $3 \mathrm{~d}$ group compared to the 1 week group.

\section{Comparisons Between Groups}

Almost two-thirds of patients who died within $7 \mathrm{~d}$ of discharge were male, reflecting the ratio of men and women who die by suicide in the UK. There were no differences in the sociodemographic features between the two groups (Table 1).

The most common primary diagnosis in both groups was affective disorder (bipolar disorder and depression). Those who died in the first $3 \mathrm{~d}$ were more likely than later post-discharge deaths to have a diagnosis of personality disorder. Patients that died within $3 \mathrm{~d}$ post-discharge and had a diagnosis of a personality disorder were also more likely to have an additional secondary diagnosis $(19,29.2 \%$ v. $46,70.8 \%$, $\left.\chi^{2}=5.49, p=.02\right)$, most commonly depressive illness $(15,32.6 \%)$. Clinical features were similar in both groups, with high rates of previous self-harm, recent adverse life events, and a short $(<7 \mathrm{~d})$ last admission. However, those who died in the first $3 \mathrm{~d}$ were more likely than those who died later in the week to have initiated their own discharge. Further, patients in the $3 \mathrm{~d}$ group were more likely to die before the follow-up appointment $\left(238,64.2 \%\right.$ v. $\left.149,41.1 \%, \chi^{2}=39.29, \mathrm{p}<.01\right)$ or to have no follow-up appointment arranged $(49,11.9 \%$ v. 29 , $7.5 \%, \chi^{2}=4.38, \mathrm{p}=.04$ ). For all patients who died following discharge, the most common method of suicide was hanging/ strangulation. Nearly a quarter of patients in the $3 \mathrm{~d}$ group died by jumping from a height or in front of a moving vehicle, significantly more than later post-discharge deaths $(23.8 \% \mathrm{v}$. $\left.16.3 \%, \chi^{2}=7.19, p<.01\right)$.

\section{DISCUSSION}

\section{Main Findings}

We found the characteristics of patients who died by suicide in the first few days after discharge to include a primary diagnosis of affective disorder (24), patient-initiated discharge (16), and 


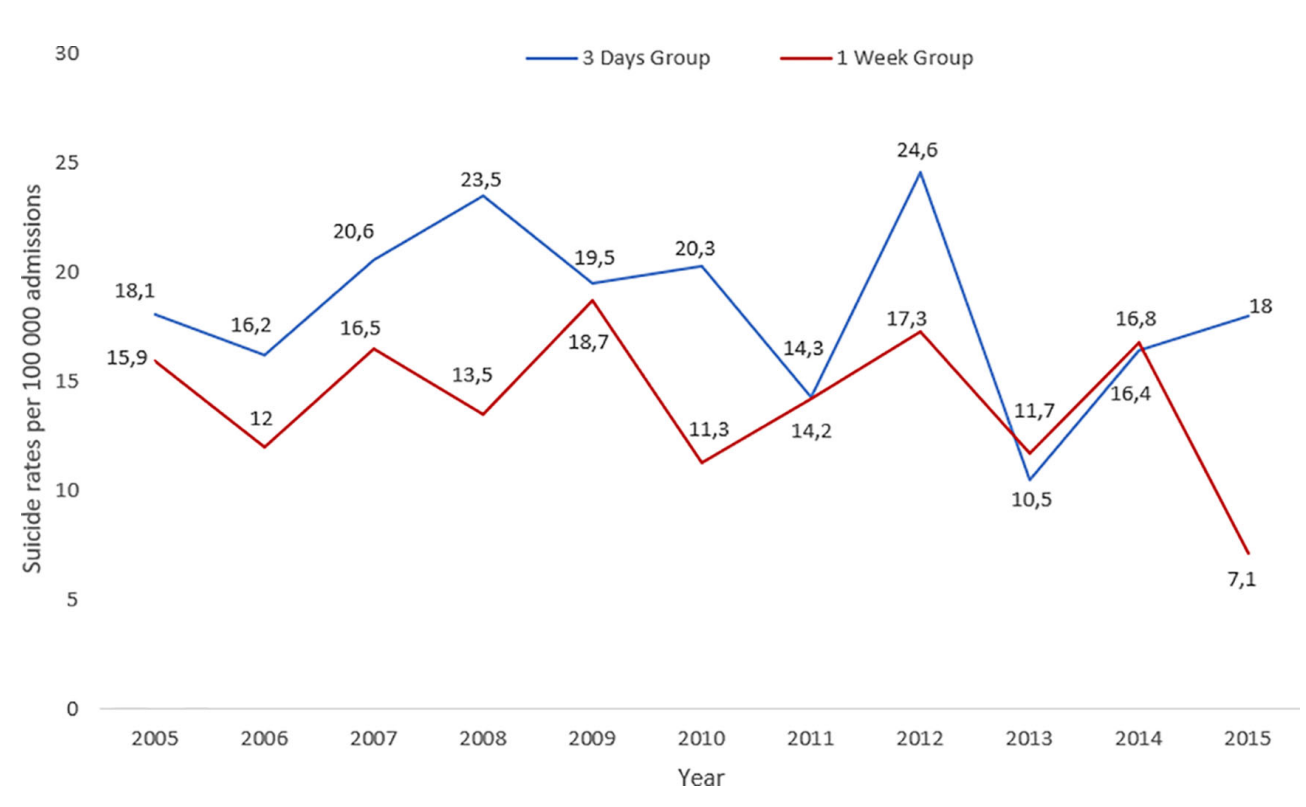

FIGURE 2 | Rates of suicide per 100,000 admissions to psychiatric in-patient care per year in England and Wales between 1st January 1997 until 31 st December 2015 for $3 \mathrm{~d}$ and 1 week group.

hanging/strangulation as the most common suicide method (4). These fit well with the general characteristics of post-discharge suicides reported in other studies $(1,4,10,11)$.

It is important to note that patients in the $3 \mathrm{~d}$ group were more likely to die before follow-up compared to 1 week group. This implies that following a more stringent follow up time frame has the potential to save lives. A diagnosis of personality disorder was significantly more common in the $3 \mathrm{~d}$ group compared to patients in the 1 week group. The lack of clear pathways into care and referral options for patients with personality disorders may present an additional challenge in the care of these patients (25). This may be especially challenging at the time of discharge, both for patients and clinicians, and could lead to patients with personality disorder being discharged

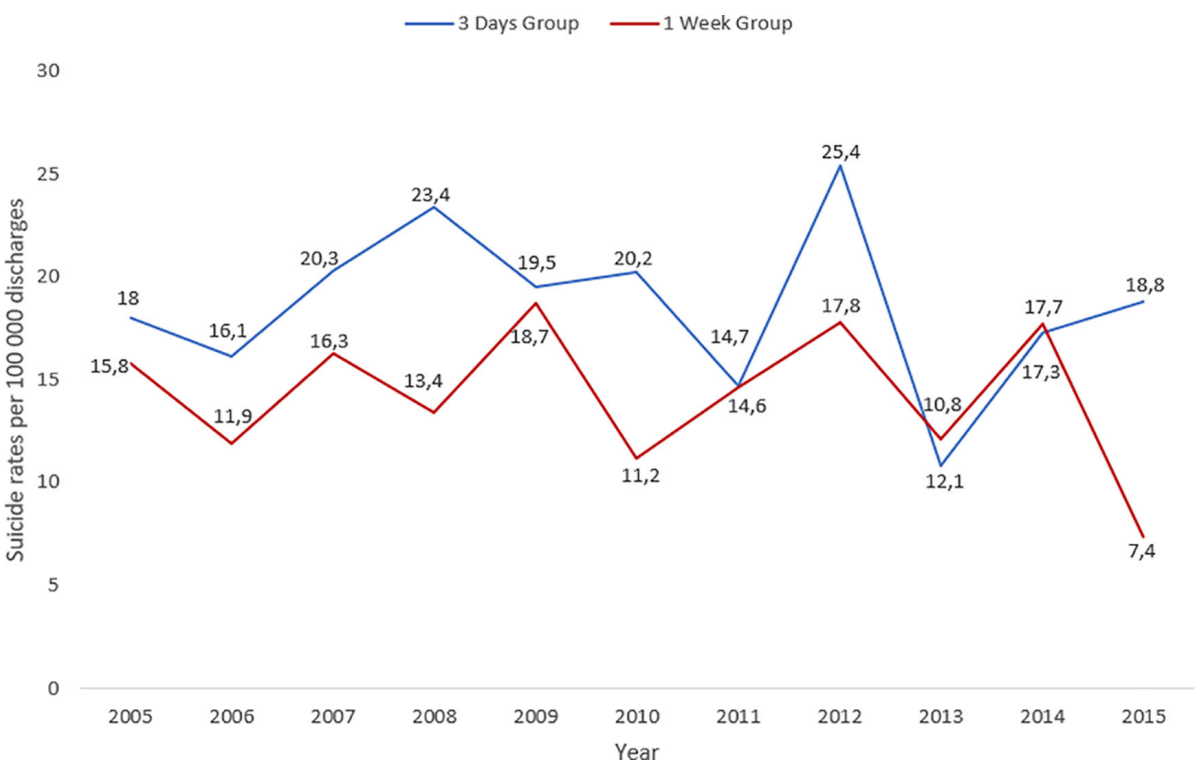

FIGURE 3 | Rates of suicide per 100,000 discharges from psychiatric in-patient care per year in England and Wales between 1st January 1997 until 31 st December 2015 for $3 d$ and 1 week group. 
TABLE 1 | Sociodemographic and clinical characteristics of patients who died within a week post-discharge $(N=826)$.

\begin{tabular}{|c|c|c|c|c|}
\hline Patient characteristic & $\begin{array}{c}3 \text { Days Group } \\
N=428 \\
\text { n (valid \%) }\end{array}$ & $\begin{array}{c}1 \text { Week Group } \\
N=398 \\
\text { n (valid \%) }\end{array}$ & $\chi^{2}$ & $p$ \\
\hline \multicolumn{5}{|l|}{ Socio-demographics } \\
\hline Male & $280(65.4)$ & $261(65.6)$ & $<.01$ & .96 \\
\hline Black, Asian and minority ethnic group & $20(4.8)$ & $22(5.6)$ & .28 & .60 \\
\hline Unmarried & $313(73.8)$ & $272(68.9)$ & 2.47 & .12 \\
\hline Unemployed & $192(45.0)$ & $173(44.5)$ & .02 & .89 \\
\hline \multicolumn{5}{|l|}{ Suicide method } \\
\hline Hanging/strangulation & $158(36.9)$ & $158(39.7)$ & .68 & .41 \\
\hline Self-poisoning & $79(18.5)$ & $87(21.9)$ & 1.49 & .22 \\
\hline Drowning & $32(7.5)$ & $32(8.0)$ & .09 & .76 \\
\hline Jumping from a height/in front of a vehicle & $102(23.8)$ & 65 (16.3) & 7.19 & $<.01^{\star \star}$ \\
\hline Other method ${ }^{1}$ & $40(9.4)$ & $34(8.5)$ & .16 & .69 \\
\hline Personality disorder & $65(15.3)$ & $37(9.3)$ & 6.81 & $<.01^{* *}$ \\
\hline Other primary diagnosis ${ }^{2}$ & $63(14.9)$ & $64(16.1)$ & .25 & .62 \\
\hline Any secondary diagnosis & $244(57.6)$ & $205(51.8)$ & 2.76 & .10 \\
\hline \multicolumn{5}{|l|}{ Clinical characteristics } \\
\hline Duration of last admission $<7 \mathrm{~d}$ & $162(38.2)$ & $140(35.3)$ & .76 & .38 \\
\hline Last admission was a re-admission & $112(27.0)$ & $93(23.5)$ & 1.32 & .25 \\
\hline Last discharge was self-discharge & $72(17.1)$ & $41(10.5)$ & 7.48 & $.02^{*}$ \\
\hline Non-adherent to medication in last month prior to death & $55(13.1)$ & $49(12.8)$ & .02 & .90 \\
\hline Any adverse life event in preceding 3 months & 219 (53.3) & $205(53.3)$ & $<.01$ & .99 \\
\hline No follow-up appointment arranged & $49(11.9)$ & $29(7.5)$ & 4.38 & $.04^{*}$ \\
\hline Follow-up arranged within 3 d post-discharge & $157(46.3)$ & $116(38.5)$ & 3.94 & .05 \\
\hline Follow-up arranged within a week post-discharge & $252(74.3)$ & $239(79.4)$ & 2.29 & .13 \\
\hline Suicide occurred before follow-up appointment & $238(64.2)$ & $149(41.1)$ & 39.3 & $<.01^{\star \star}$ \\
\hline
\end{tabular}

${ }^{*} p<.05,{ }^{* *} p<.01,{ }^{1}$ other methods of suicide include firearms, cutting/stabbing, burning, electrocution, suffocation/asphyxiation, and cO poisoning. ${ }^{2}$ other primary diagnoses include adjustment disorder, anxiety disorders, organic disorders, dementia, eating disorders, ADHD/conduct disorder, somatoform/somatisation disorder, and pervasive development disorder.

${ }^{3}$ Estimation of risk was provided by the clinician filling out the questionnaire.

into services that may not be able to provide the specialist expertise recommended by the National Institute for Health and Care Excellence (NICE) guidance (25). The finding that patients in the $3 \mathrm{~d}$ group had initiated their own discharge more often than patients in the 1 week group is important. Selfdischarge and a short final admission have been frequently cited in the literature as being associated with suicide $(4,5,7$, 15-17). Meehan and colleagues (5) acknowledged that patients who initiate their own discharge are by definition harder to engage with services after leaving hospital. A self-initiated discharge may indicate a lack of engagement with services, as well as more severe illness compared to patients who have been assessed as well-enough to transfer from in-patient to community care. Finally, patients in the $3 \mathrm{~d}$ group were more likely to die by jumping from a height or in front of a moving vehicle than those in the 1 week group. A previous study of
NCISH data (26) found suicide by jumping a more common cause of death in inpatients and recently discharged patients than in the general population, hypothesizing the availability of the method and a lack of access to alternative methods as the most likely contributory factor. People who attempt suicide by jumping have often been described as ambivalent and if they are derailed from their attempt do not subsequently die by suicide (27).

On the whole, rates of patients who die in the early postdischarge period were small and fluctuated considerably over the study period. There was a peak in 2012, consistent with an overall increase in suicide by recently discharged patients that has since declined (2). In the last year with complete data (2015) there was an evident discrepancy between suicide rates in the $3 \mathrm{~d}$ and 1 week group, with the 1 week group showing a fall. However, due to low numbers per year, an in-depth analysis was not possible. 


\section{Methodological Issues}

This is, to the authors' knowledge, the first study describing the characteristics of patients who died by suicide within two recommended follow-up periods post-discharge. However, there are some methodological limitations. As this is an exploratory, uncontrolled retrospective study, we cannot make causal inferences. Established limitations of NCISH data include the inability to draw etiological conclusions and the potential bias of the clinicians providing information (28). It is possible that some deaths by suicide may have been missed, however this is minimized by including deaths classified as both suicide and open verdict. Diagnoses were made by the treating clinician and we are unsure which tools were used during the diagnostic process. Of concern for this study in particular is the grouping of different types of personality disorders into one variable where the clinical picture for each disorder might differ. Additionally, the lack of information on the hour of patients' discharge and death has prevented us from having a clearer picture of the time that had passed between the death and discharge and in return to place patients into post-discharge groups more precisely. For rates, numbers of post-discharge suicides per year were too small to allow for a meaningful time-series analysis. Finally, due to incompleteness, 2016 data could not be included in our rates analysis.

\section{Implications for Future Research}

The comparison of patients who died by suicide in two recent post-discharge periods presents novel information with practical implications for discharge and safety planning. There is need for further research into discharge planning, both of a quantitative and qualitative nature. Specifically, more research is needed on the discharge needs of patients with different types of personality disorder (e.g. borderline, avoidant). Clinicians should be aware of the increased risk of suicide in the immediate post-discharge period by people with a diagnosis of personality disorder and in those who have initiated their own discharge, as well as the increased risk of jumping from a height or in front of vehicle shortly after discharge. This information should be incorporated into discharge and safety planning in collaboration with the patients and their families or carers where possible, including an assessment of the patient's social support. Current UK guidance is for $7 \mathrm{~d}$ follow-up after discharge from in-patient care. The current findings suggest that deaths occurring immediately following discharge involve more highly lethal and immediately-available methods. Our findings support the recent recommendation from NCISH that follow-up should occur within $3 \mathrm{~d}$ of discharge from in-patient care (2). In

\section{REFERENCES}

1. Chung D, Hadzi-Pavlovic D, Wang M, Swaraj S, Olfson M, Large M. Metaanalysis of suicide rates in the first week and the first month after psychiatric hospitalisation. BMJ Open (2019) 9(3):e023883. doi: 10.1136/bmjopen-2018023883

2. National Confidential Inquiry into Suicide and Safety in Mental Health. Annual Report. England, Northern Ireland, Scotland: Wales. University of Manchester; (2018). addition, the data suggests this should be done as soon as possible-in some cases preferably on the very first postdischarge day.

\section{DATA AVAILABILITY STATEMENT}

The datasets generated for this study will not be made publicly available: Datasets contain personal details of patients that died by suicide.

\section{ETHICS STATEMENT}

The studies involving human participants were reviewed and approved by North West Research Ethical Committee. Written informed consent for participation was not required for this study in accordance with the national legislation and the institutional requirements.

\section{AUTHOR CONTRIBUTIONS}

All authors contributed to the study conception and design. Material preparation and analysis were performed by LB. The first draft of the manuscript was written by LB and all authors commented on previous versions of the manuscript. All authors read and approved the final manuscript.

\section{FUNDING}

This research was supported by the Healthcare Quality Improvement Partnership (HQIP NCA 2069, Mental Health Clinical Outcome Review Programme). The study was carried out as part of the National Confidential Inquiry into Suicide and Safety in Mental Health.

\section{ACKNOWLEDGMENTS}

We thank the other members of the research team: James Burns, Huma Daud, Jane Graney, Julie Hall, Saied Ibrahim, Rebecca Lowe, Nicola Richards, Cathryn Rodway, Phil Stones, Prof Jennifer Shaw, Su-Gwan Tham, and Nicola Worthington.

3. Chung DT, Ryan CJ, Hadzi-Pavlovic D, Singh SP, Stanton C, Large MM Suicide Rates After Discharge From Psychiatric Facilities: A Systematic Review and Meta-analysis. JAMA Psychiatry (2017) 74(7):694. doi: 10.1001/ jamapsychiatry.2017.1044

4. Hunt IM, Kapur N, Webb R, Robinson J, Burns J, Shaw J, et al. Suicide in recently discharged psychiatric patients: a case-control study. Psychol Med (2009) 39(3):443-9. doi: 10.1017/S0033291708003644

5. Meehan J, Kapur N, Hunt IM, Turnbull P, Robinson J, Bickley H, et al. Suicide in mental health in-patients and within 3 months of discharge: National 
clinical survey. Br J Psychiatry (2006) 188(2):129-34. doi: 10.1192/ bjp.188.2.129

6. Bickley H, Hunt IM, Windfuhr K, Shaw J, Appleby L, Kapur N. Suicide Within Two Weeks of Discharge From Psychiatric Inpatient Care: A CaseControl Study. Psychiatr Serv. (2013) 64(7):653-9. doi: 10.1176/ appi.ps.201200026

7. Riblet N, Shiner B, Watts BV, Mills P, Rusch B, Hemphill RR. Death by Suicide Within 1 Week of Hospital Discharge: A Retrospective Study of Root Cause Analysis Reports. J Nerv. Ment Dis (2017) 205(6):436-42. doi: 10.1097/ NMD.0000000000000687

8. Deisenhammer EA, Huber M, Kemmler G, Weiss EM, Hinterhuber H. Psychiatric hospitalizations during the last 12 months before suicide. Gen Hosp. Psychiatry (2007) 29(1):63-5. doi: 10.1016/j.genhosppsych.2006.09.007

9. National Institute for Health and Care Excellence. Transition between inpatient mental health settings and community or care home settings. 2016. (NICE Guideline). Report No.: [NG53].

10. Pirkola S, Sohlman B, Wahlbeck K. The characteristics of suicides within a week of discharge after psychiatric hospitalisation - a nationwide register study. BMC Psychiatry (2005) 5(1):32. doi: 10.1186/1471-244X-5-32

11. Troister T, Links PS, Cutcliffe J. Review of predictors of suicide within 1 year of discharge from a psychiatric hospital. Curr Psychiatry Rep (2008) 10(1):605. doi: 10.1007/s11920-008-0011-8

12. Kan C-K, Ho T-P, Dong JYS, Dunn ELW. Risk factors for suicide in the immediate post-discharge period. Soc Psychiatry Psychiatr Epidemiol. (2007) 42(3):208-14. doi: 10.1007/s00127-006-0153-0

13. Crawford MJ. Suicide following discharge from in-patient psychiatric care. Adv Psychiatr Treat (2004) 10(6):434-8. doi: 10.1192/apt.10.6.434

14. Haglund A, Lysell H, Larsson H, Lichtenstein P, Runeson B. Suicide Immediately After Discharge From Psychiatric Inpatient Care: A Cohort Study of Nearly 2.9 Million Discharges. J Clin Psychiatry (2019) 80(2):e1-6. doi: $10.4088 / \mathrm{JCP} .18 \mathrm{~m} 12172$

15. Riblet N, Richardson JS, Shiner B, Peltzman TR, Watts BV, McCarthy JF. Death by Suicide in the First Year After Irregular Discharge From Inpatient Hospitalization. Psychiatr Serv. (2018) 69(9):1032-5. doi: 10.1176/ appi.ps.201800024

16. Qin P, Nordentoft M. Suicide Risk in Relation to Psychiatric Hospitalization: Evidence Based on Longitudinal Registers. Arch Gen Psychiatry (2005) 62 (4):427. doi: 10.1001/archpsyc.62.4.427

17. Lin H-C, Lee H-C, Kuo N-W, Chu C-H. Hospital characteristics associated with post-discharge suicide of severely depressed patients. J Affect Disord (2008) 110(3):215-21. doi: 10.1016/j.jad.2008.01.030

18. Tseng M-CM, Chang C-H, Liao S-C, Yeh Y-C. Length of stay in relation to the risk of inpatient and post-discharge suicides: A national health insurance claim data study. J Affect Disord (2020) 266:528-33. doi: 10.1016/ j.jad.2020.02.014

19. Qurashi I, Kapur N, Appleby L. A Prospective Study of Noncompliance With Medication, Suicidal Ideation, and Suicidal Behavior in Recently Discharged
Psychiatric Inpatients. Arch Suicide Res (2006) 10(1):61-7. doi: 10.1080/ 13811110500318455

20. Links P, Nisenbaum R, Ambreen M, Balderson K, Bergmans Y, Eynan R, et al. Prospective study of risk factors for increased suicide ideation and behavior following recent discharge. Gen Hosp. Psychiatry (2012) 34(1):88-97. doi: 10.1016/j.genhosppsych.2011.08.016

21. Appleby L, Shaw J, Sherratt J, Amos T, Robinson J, McDonnell R, et al. Safety First: Five-year Report of the National Confidential Inquiry into Suicide and Homicide by People with Mental Illness. London: UK Department of Health; (2001).

22. StataCorp. Stata Statistical Software. College Station, Texas: Statacorp Llc; (2017).

23. RStudio Team. RStudio: Integrated Development for R. Boston, MA: RStudio, Inc. (2016). Available from: http://www.rstudio.com/.

24. Olfson M, Wall M, Wang S, Crystal S, Liu S-M, Gerhard T, et al. Short-term Suicide Risk After Psychiatric Hospital Discharge. JAMA Psychiatry (2016) 73 (11):1119. doi: 10.1001/jamapsychiatry.2016.2035

25. National Confidential Inquiry into Suicide and Safety in Mental Health. Safer Care for Patients with Personality Disorder. Manchester: University of Manchester; (2018).

26. Hunt IM, Swinson N, Palmer B, Turnbull P, Cooper J, While D, et al. Method of Suicide in the Mentally Ill: A National Clinical Survey. Suicide Life Threat Behav (2010) 40(1):22-34. doi: 10.1521/suli.2010.40.1.22

27. Beautrais A. Suicide by Jumping: A Review of Research and Prevention Strategies. Crisis (2007) 28(S1):58-63. doi: 10.1027/0227-5910.28.S1.58

28. Appleby L, Shaw J, Amos T, McDonnell R, Harris C, McCann K, et al. Suicide within 12 months of contact with mental health services: national clinical survey. BMJ (1999) 318(7193):1235-9. doi: 10.1136/bmj.318.7193.1235

Conflict of Interest: LA chairs the Suicide Prevention Advisory Group at the Department of Health and is a non-executive Director for the Care Quality Commission. NK is a member of the Suicide Prevention Advisory Group. NK also chaired the guideline development group for the 2012 NICE guidelines on the longer-term management of self-harm and currently chairs the guideline development group for the NICE depression in adults guideline.

The remaining authors declare that the research was conducted in the absence of any commercial or financial relationships that could be construed as a potential conflict of interest.

Copyright (๑ 2020 Bojanić, Hunt, Baird, Kapur, Appleby and Turnbull. This is an open-access article distributed under the terms of the Creative Commons Attribution License (CC BY). The use, distribution or reproduction in other forums is permitted, provided the original author(s) and the copyright owner(s) are credited and that the original publication in this journal is cited, in accordance with accepted academic practice. No use, distribution or reproduction is permitted which does not comply with these terms. 